\title{
Plasmonic band-edge modulated surface-enhanced Raman scattering
}

Lie-rong Yuan, Kang Qin, Jun Tan, Peng Bao, Guo-xin Cui, Qian-jin Wang, Stephen D. Evans, Yan-qing Lu, Yong-yuan Zhu, and Xue-jin Zhang

Citation: Appl. Phys. Lett. 111, 051601 (2017); doi: 10.1063/1.4997303

View online: http://dx.doi.org/10.1063/1.4997303

View Table of Contents: http://aip.scitation.org/toc/apl/111/5

Published by the American Institute of Physics

\section{Articles you may be interested in}

A three-dimensional all-metal terahertz metamaterial perfect absorber Applied Physics Letters 111, 051101 (2017); 10.1063/1.4996897

Active tuning of high- $Q$ dielectric metasurfaces

Applied Physics Letters 111, 053102 (2017); 10.1063/1.4997301

Structural and electrical analysis of epitaxial 2D/3D vertical heterojunctions of monolayer $\mathrm{MoS}_{2}$ on $\mathrm{GaN}$ Applied Physics Letters 111, 051602 (2017); 10.1063/1.4997188

Measuring microwave cavity response using atomic Rabi resonances

Applied Physics Letters 111, 051103 (2017); 10.1063/1.4997302

Surface plasmon enhanced Si-based BIB terahertz detectors

Applied Physics Letters 111, 053505 (2017); 10.1063/1.4996496

Ultra-broadband graphene-InSb heterojunction photodetector

Applied Physics Letters 111, 051106 (2017); 10.1063/1.4997327

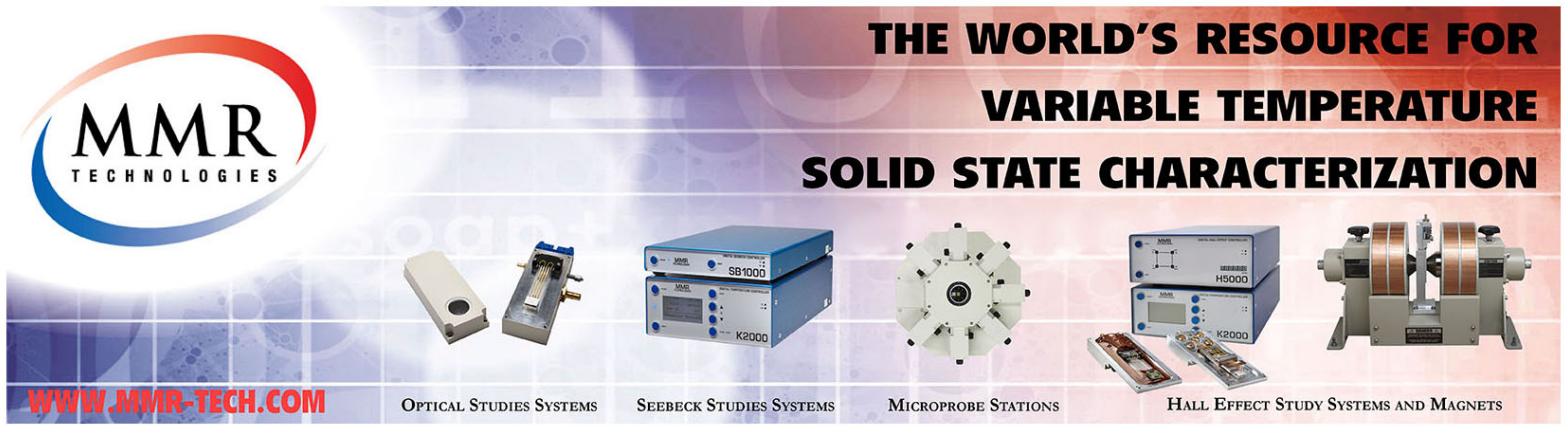




\title{
Plasmonic band-edge modulated surface-enhanced Raman scattering
}

\author{
Lie-rong Yuan, ${ }^{1,2}$ Kang Qin, ${ }^{1,3}$ Jun Tan, ${ }^{1,2}$ Peng Bao, ${ }^{4}$ Guo-xin Cui, ${ }^{1,5}$ Qian-jin Wang, ${ }^{1,5}$ \\ Stephen D. Evans, ${ }^{4}$ Yan-qing Lu, ${ }^{1,5, a)}$ Yong-yuan Zhu, ${ }^{1,2, b)}$ and Xue-jin Zhang ${ }^{1,5, c)}$ \\ ${ }^{1}$ National Laboratory of Solid State Microstructures and Collaborative Innovation Center of Advanced \\ Microstructures, Nanjing University, Nanjing 210093, China \\ ${ }^{2}$ School of Physics, Nanjing University, Nanjing 210093, China \\ ${ }^{3}$ Kuang Yaming Honors School, Nanjing University, Nanjing 210093, China \\ ${ }^{4}$ School of Physics and Astronomy, University of Leeds, Leeds LS2 9JT, United Kingdom \\ ${ }^{5}$ College of Engineering and Applied Sciences, Nanjing University, Nanjing 210093, China
}

(Received 1 April 2017; accepted 22 July 2017; published online 2 August 2017)

\begin{abstract}
The band structure of surface plasmon polaritons (SPPs) on the Ag surface in the presence of gratings and SPP-based surface-enhanced Raman scattering (SERS) are investigated theoretically and experimentally. The SPP bandgap position can be tuned by geometric parameters. The SPP band edge dominates the SERS behavior. The template stripping process is introduced to reduce SPP propagation losses, improving SERS sensitivity by $\sim 40$. Apart from flexibility and a moderate SERS enhancement factor of the order of $10^{5}-10^{6}$, the SPP band structure is highly reproducible with a relative standard deviation of $10.9 \%$. Our results open opportunities for SPP band structures to serve as SERS substrates. Published by AIP Publishing. [http://dx.doi.org/10.1063/1.4997303]
\end{abstract}

Surface-enhanced Raman scattering (SERS) is a powerful spectroscopic technique for chemical and biological analyses, for identifying the chemical fingerprint of the molecules. ${ }^{1}$ The physical mechanism that dominates the enhancement behavior is based on strong electric fields associated with localized surface plasmon resonances (LSPRs). Recent SERS systems have been predominantly based on dimer structures, i.e., nanoparticle pairs of noble metals or particle on mirror configurations. ${ }^{2-4}$ Large amplification of an electrical field occurs in the gap of a dimer structure compared to that at the surface of a single nanoparticle. The gap positions are termed SERS "hot spots". Using such plasmonically enhanced spectroscopy, the Raman detection of single molecules has become a reality. ${ }^{5-8}$ High sensitivity SERS systems often suffer from variable signal magnitudes, making their use as quantitative metrological techniques problematic, and thus, there is a demand for SERS substrates which can show reproducible signal enhancement and can be manufactured easily. Periodically arranged "hot spots" such as nanogap array structures would be of benefit to attain this goal. ${ }^{9-14}$ In this situation, the collective effect of the periodic arrangement of nanostructures often emerges. For instance, "hot spots" could be linked with surface plasmon polaritons (SPPs) and interact with each other, which can be utilized to supplement the Raman enhancement factor (EF). However, the surface roughness and inhomogeneities will reduce the device performance as the SPP is extremely sensitive to the support surface or interface. ${ }^{15}$

In this letter, we investigate the impact of SPPs, rather than LSPRs, on the SERS properties, taking a simple onedimensional (1-D) grating structure as an example. The grating parameters are optimized with regard to Raman EF. Both the theoretical and experimental results show that the SPP

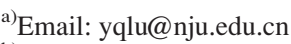

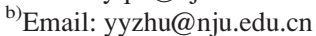

${ }^{c)}$ Email: xuejinzh@nju.edu.cn
}

band edge plays an overwhelming role in the field enhancement. In our experiments, the 1-D grating structure was formed on ultrasmooth, template stripped Ag films thus minimizing SPP propagation losses and the reproducibility of such a SERS substrates evaluated.

The template stripping process was adopted to obtain an ultrasmooth $\mathrm{Ag}$ surface by means of a cleaned $\mathrm{Si}$ substrate, in which gratings were fabricated by focused ion beam (FIB) etching (strata FIB 201, FEI Co. $30 \mathrm{keV} \mathrm{Ga} \mathrm{ions).} \mathrm{Then,} \mathrm{a}$ $200 \mathrm{~nm}$ Ag film was deposited on the Si substrate using ion beam sputtering at a rate of $6.0 \mathrm{~nm} / \mathrm{min}$ under $7 \mathrm{keV}$ and $300 \mu \mathrm{A}$. After that, a $\sim 1 \mathrm{~mm}$ thick $\mathrm{Cu}$ foil was electrodeposited on the $\mathrm{Ag}$ film and peeled off from the $\mathrm{Si}$ substrate together with the patterned Ag film. The fabrication process is illustrated in Fig. 1(a). The surface morphology of stripped surface of the $\mathrm{Ag}$ film was characterized by atomic force microscopy (AFM). A three-dimensional (3-D) AFM image of $\mathrm{Ag}$ grating is shown in Fig. 1(b). The root-mean-square roughness of the $\mathrm{Ag}$ surface with and without the template stripping process is 0.980 and $2.72 \mathrm{~nm}$, respectively, over an area of $2 \times 2 \mu \mathrm{m}^{2}$.

The structural parameter optimization and the theoretical SPP band structure of the 1-D grating were investigated using 3-D finite-difference time-domain (FDTD) software (Lumerical FDTD Solutions). The Raman electromagnetic $\mathrm{EF}$ approximately scales as $g^{4}, g=|E| /\left|E_{0}\right|$, where $E$ is the resulting electric field outside the 1-D grating and $E_{0}$ is the incident electric field. There are three adjustable structural parameters for a 1-D rectangular grating, i.e., the grating period $(P)$, the groove depth $(H)$, and the ridge width $(W)$, as depicted in Fig. 1(a). The largest electric field intensity, for an incident wavelength of $\lambda=532 \mathrm{~nm}$, was determined by scanning the above parameters. The Fabry-Pérot (F-P) cavity resonance within the grating grooves results in discrete extreme values of the groove depth, when the other two parameters are fixed. For simplicity, we set the groove depth $H=30 \mathrm{~nm}$, corresponding to the first-order F-P resonance. In 
(a)

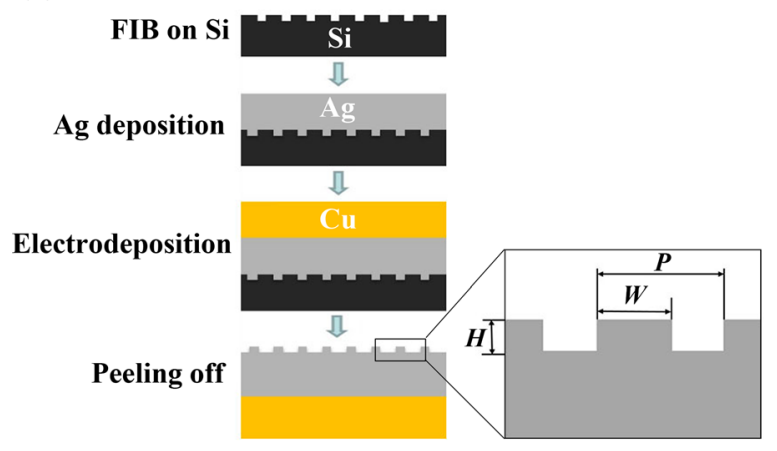

(b)

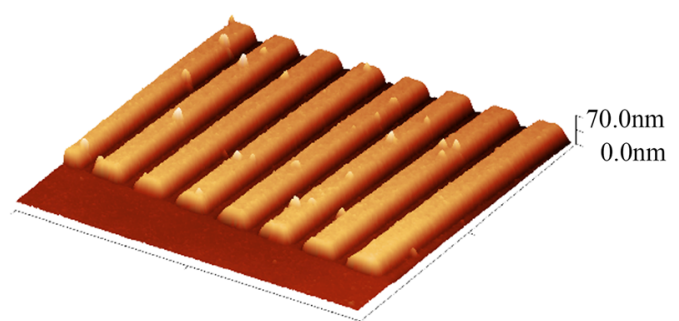

FIG. 1. (a) Schematic diagram of the template stripping process. The enlargement shows a cross-sectional sketch of the 1-D grating. (b) The 3-D AFM image of the grating.

this case, the electromagnetic $\mathrm{EF}$ depends on period $P$ and the duty ratio $(r=W / P)$. Figure 2 presents the mapping image of the calculated electromagnetic EF, from which the optimum values of $P$ and $r$ can be determined as $475 \mathrm{~nm}$ and 0.57 , respectively.

It is well known that the size of an SPP bandgap changes with $r$ and $H$, while its central position changes with $P$. Here, we demonstrate the influence of $P$ on the SPP band structure with $H=30 \mathrm{~nm}$ and $r=0.57$. From the calculated dispersion relation shown in Fig. 3(a), one can see that the bandgap is opened up, and the central bandgap position red shifts with $P$. Especially, the upper and lower SPP band edges lie in $\lambda=532 \mathrm{~nm}$ for $P=475$ and $525 \mathrm{~nm}$, respectively. The experimental dispersion relations were obtained by mapping the reflectivity in a plane conjugate to the Fourier (back focal) plane of a $50 \times$ objective. The reflectivity indicates whether SPP modes are generated or not. The SPPs are excited at

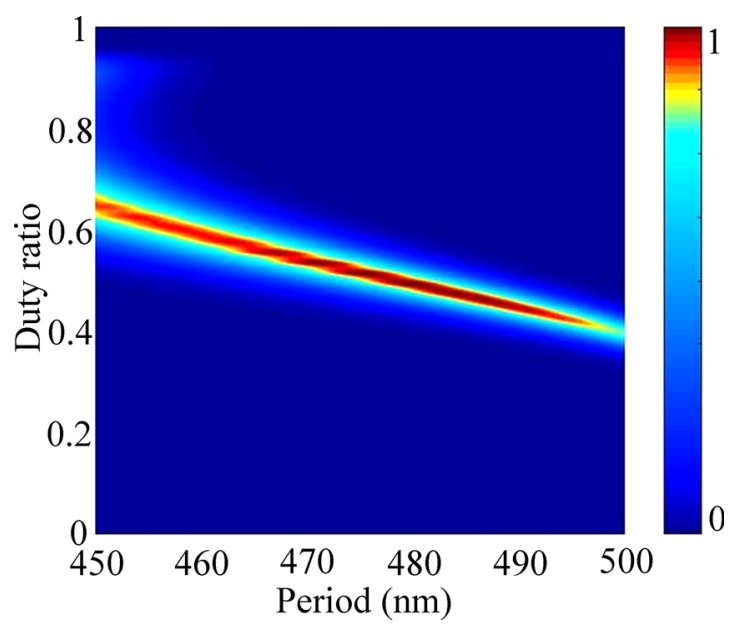

FIG. 2. The calculated EF as a function of the grating period and the duty ratio.
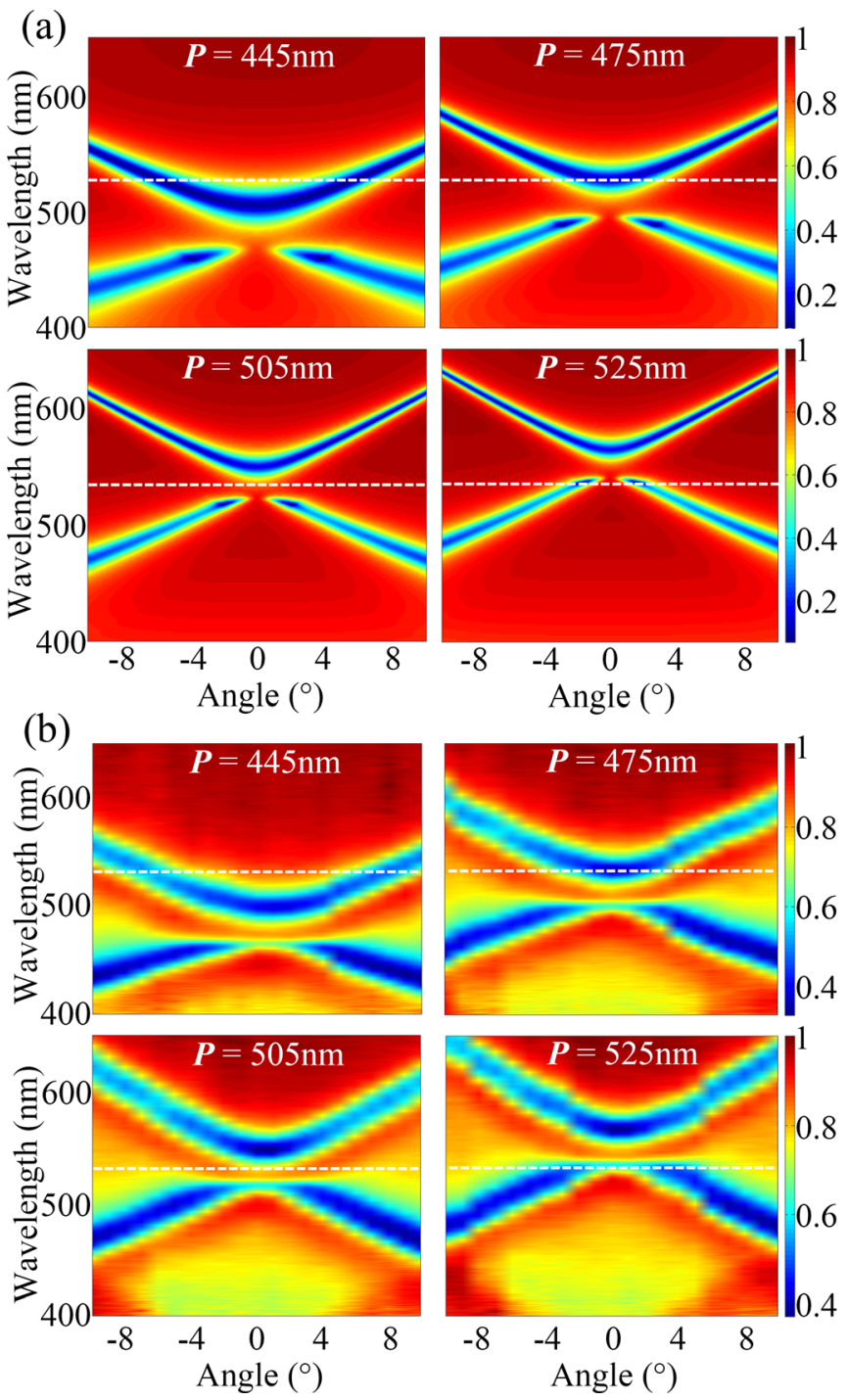

FIG. 3. (a) The theoretical and (b) experimental dispersion relation of the 1-D gratings with various periods but the same groove depth $H=30 \mathrm{~nm}$ and duty ratio $r=0.57$. The white dashed line corresponds to the position of incident wavelength $\lambda=532 \mathrm{~nm}$.

certain angles and wavelengths with lower reflectivities. Figure 3(b) shows the measured dispersion relations, which are in good agreement with those calculated. Compared to the theoretical one, the measured lower SPP band edge is not clearly distinguished partially due to the limited angular resolution of $1^{\circ}$ in the experiments.

For a grating, the resultant electric field is inversely proportional to SPP group velocity $v_{\mathrm{g}}=d \omega / d k{ }^{16}$ The smaller the $v_{\mathrm{g}}$, the larger the magnitude of $E$. From Fig. 3, the smallest SPP group velocity $v_{\mathrm{g}}$ locates at the SPP band edge. As a far-field property, the reflectivity provides valid information on the near-field property. The values of reflectivity near zero angle at $\lambda=532 \mathrm{~nm}$ were extracted for different periods, as is shown in Fig. 4(a). It can be seen that the trend of the theoretical curve is coincident with that of the experimental curve. Two valleys of reflectivity are in line with the upper and lower SPP band edges, respectively. We calculated the electric field at $\lambda=532 \mathrm{~nm}$ for different $P$ values, as shown in Fig. 4(b). The maximum $|E|$ appears with minimum reflectivity. Both the minimal reflectivity and the strongest electric 


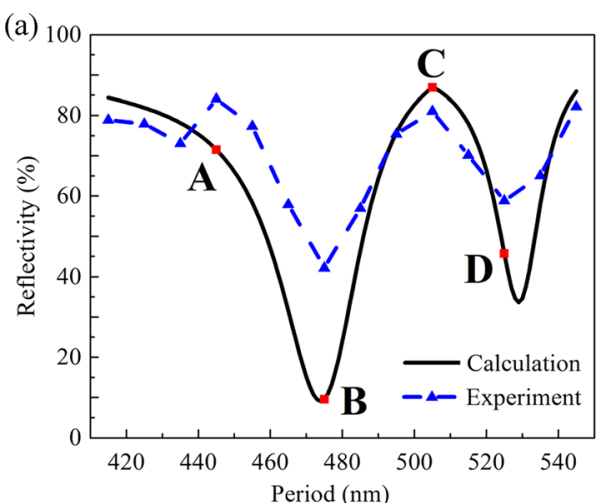

(b)
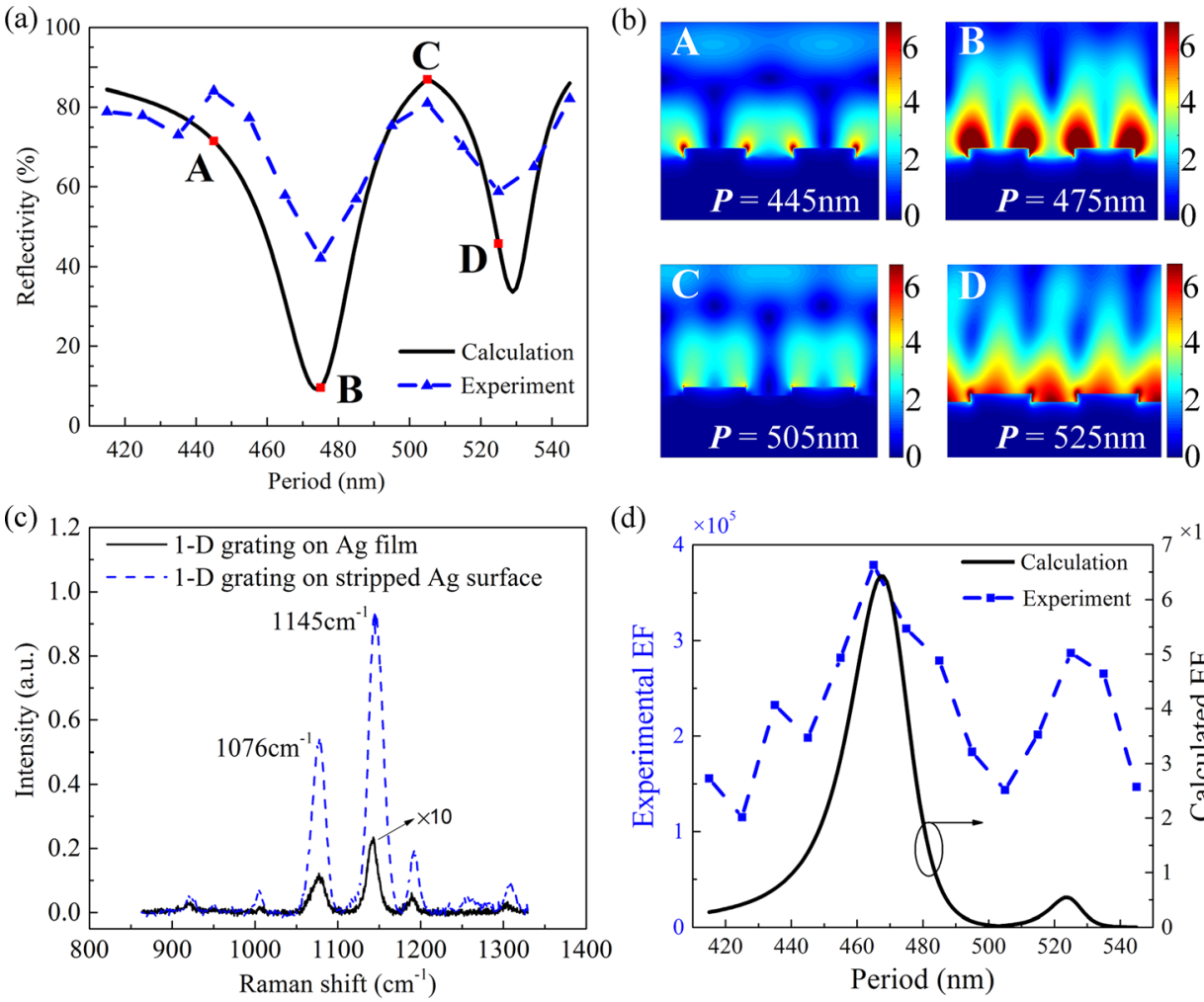

(d)

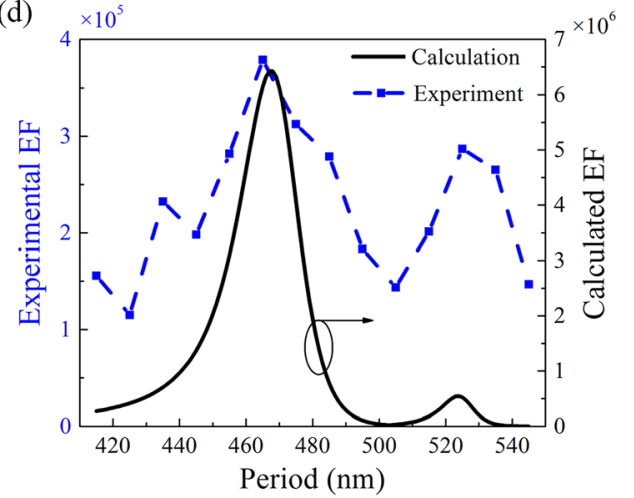

FIG. 4. (a) Theoretical and experimental reflectivity at $\lambda=532 \mathrm{~nm}$ for the 1D gratings with various periods but the same groove depth $H=30 \mathrm{~nm}$ and duty ratio $r=0.57$. (b) The electric field distribution at $\lambda=532 \mathrm{~nm}$ of four representative grating periods. (c) Raman spectra taken from the same 1-D grating structure with $H=30 \mathrm{~nm}$, $r=0.57$ and $P=475 \mathrm{~nm}$ on the $\mathrm{Ag}$ surface with/without the template stripping process, as illustrated in Fig. 1(a). (d) The SERS EF at the $1145 \mathrm{~cm}^{-1}$ corresponding to (a). field occur at $P=475 \mathrm{~nm}$ (position $B$ ), conforming with the results of Figs. 2 and 3. The electric field is weakest at $P=505 \mathrm{~nm}$ (position $C$ ) due to the fact that $\lambda=532 \mathrm{~nm}$ is within the SPP bandgap. That is, no SPP modes exist in this case, referring to the dispersion relation shown in Fig. 3. As for the SERS performance, the upper SPP band edge behaves better than the lower one, which can be observed from the SPP band structure shown in Fig. 3(a). Moreover, the electric filed distribution of 1-D gratings in Fig. 4(b) shows that the largest $|E|$ is situated in the corner of grating ridge because of the tip effect.

For the SERS measurements, the self-assembled monolayer of 4-aminothiophenol (4-ATP) molecules was formed on the Ag surface by the following process. The sample was immersed in an ethanol solution of 4-ATP at a concentration of $1 \times 10^{-4} \mathrm{M}$ for $6 \mathrm{~h}$. Then, the sample was rinsed several times with ethanol and deionized water to remove excessive molecules and dried up with cleaned nitrogen. After that, SERS measurements were taken immediately. The SERS properties were investigated using a confocal Raman system (Horiba Scientific, LabRAM HR Raman spectrometer). The sample was excited by a laser at $\lambda=532 \mathrm{~nm}$ via a $50 \times$ objective. We examined the superiority of the stripped structures over those directly fabricated on the Ag film using optimized grating parameters $H=30 \mathrm{~nm}, r=0.57$, and $P=475 \mathrm{~nm}$, with which the best SERS performance will be expected. Figure 4(c) shows such two different SERS spectra of the 4ATP molecular monolayer adsorbed on the 1-D Ag grating. By comparison, the maximum Raman intensity without the template stripping process is about 40 times smaller than that with the template stripping process. To estimate the experimental EF, the expression $\mathrm{EF}=\left(I_{\mathrm{SERS}} / N_{\mathrm{Surf}}\right) /\left(I_{\mathrm{Rs}} / N_{\mathrm{Vol}}\right)$ was used. ${ }^{17} I_{\mathrm{SERS}}$ and $I_{\mathrm{Rs}}$ are the measured Raman intensities of the self-assembled monolayer of 4-ATP on a 1-D grating surface and 4-ATP powder, which was placed on a clean glass slide for normal Raman measurements. The former belongs to the SERS measurements; the latter belongs to the normal Raman (non-SERS) measurements. $N_{\text {Surf }}$ and $N_{\text {Vol }}$ are the evaluated numbers of 4-ATP molecules involved in the SERS and normal Raman measurements. It should be noted that Raman peaks of adsorbed molecules shift with the substrates. In our experiments, the SERS peaks of $1076 \mathrm{~cm}^{-1}$ and $1145 \mathrm{~cm}^{-1}$ [Fig. 4(c)] correspond to normal Raman peaks of $1092 \mathrm{~cm}^{-1}$ and $1177 \mathrm{~cm}^{-1}$, respectively. We choose the SERS peak of $1145 \mathrm{~cm}^{-1}$ as an example below. The Raman spectra were measured for samples with different $P$ values, and the values of the SERS EF at $1145 \mathrm{~cm}^{-1}$ were collected to compare with those calculated, as shown in Fig. 4(d). There are two peaks of EF, which are consistent with two valleys of reflectivity in Fig. 4(a), as well as the SPP band edges in Fig. 3. The above results show that the electric field enhancement of the 1-D grating relies on the SPP group velocity $v_{\mathrm{g}}$, which can be modulated by SPP band engineering. Therein, the SPP band edge makes a significant contribution to the enhancement with the EF as large as $4 \times 10^{5}$ experimentally and $7 \times 10^{6}$ theoretically. The discrepancy between the experimental and theoretical results may arise from several factors, e.g., a rounding of the corners of grating ridge (in experiment compared to model) and SPP propagating losses induced by remaining roughness on the Ag surface in spite of the template stripping process.

The Raman mapping image was acquired to study the reproducibility of SERS substrates. Figure 5 presents a Raman mapping at the 4-ATP Raman peak of $1145 \mathrm{~cm}^{-1}$. A $50 \times$ objective was used with the scanning area of $15 \times 15$ $\mu \mathrm{m}^{2}$ and the total number of the scanning spots 225 . The relative standard deviation (RSD) is evaluated as low as $10.9 \%$. 


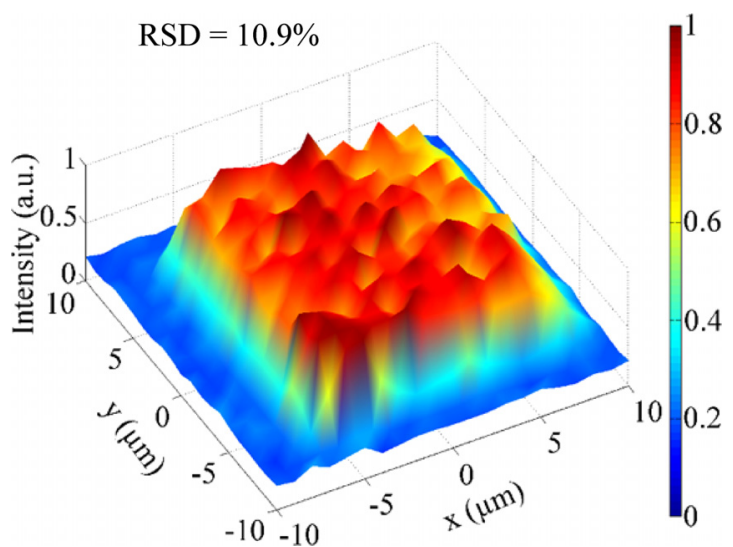

FIG. 5. SERS mapping image at $1145 \mathrm{~cm}^{-1}$ for the 1-D grating under a $50 \times$ objective.

In conclusion, we have investigated the SPP band structure of 1-D Ag gratings both theoretically and experimentally and applied it for the development of SERS substrates. The largest SERS enhancement takes place at the SPP band edge. Being identical to LSPRs, the SPP-based SERS mechanism can be ultimately linked to electromagnetic enhancement. The wavelength position of SPP band edges can be tuned by geometric parameters of gratings. The SPP-based SERS substrates have advantages of high reproducibility. The technology of template stripping is beneficial in developing SERS substrates with a large SERS EF. Owing to the flexibility and reproducibility, the SPP band structures provide a practical way for chemical and biological sensing.

This work was supported by the National Key R\&D Program of China (Grant No. 2017YFA0303700), the
National Natural Science Foundation of China (Grant Nos. 11274159 and 11374150), and by PAPD.

${ }^{1}$ Y. C. Cao, R. Jin, and C. A. Mirkin, Science 297(5586), 1536-1540 (2002).

${ }^{2}$ J. S. Huang, V. Callegari, P. Geisler, C. Bruning, J. Kern, J. C. Prangsma, X. Wu, T. Feichtner, J. Ziegler, P. Weinmann, M. Kamp, A. Forchel, P. Biagioni, U. Sennhauser, and B. Hecht, Nat. Commun. 1, 150 (2010).

${ }^{3}$ F. Benz, C. Tserkezis, L. O. Herrmann, B. de Nijs, A. Sanders, D. O. Sigle, L. Pukenas, S. D. Evans, J. Aizpurua, and J. J. Baumberg, Nano Lett. 15(1), 669-674 (2015).

${ }^{4}$ R. W. Taylor, F. Benz, D. O. Sigle, R. W. Bowman, P. Bao, J. S. Roth, G. R. Heath, S. D. Evans, and J. J. Baumberg, Sci. Rep. 4, 5940 (2014).

${ }^{5}$ S. M. Nie and S. R. Emory, Science 275(5303), 1102-1106 (1997).

${ }^{6}$ S. L. Kleinman, E. Ringe, N. Valley, K. L. Wustholz, E. Phillips, K. A. Scheidt, G. C. Schatz, and R. P. Van Duyne, J. Am. Chem. Soc. 133(11), 4115-4122 (2011).

${ }^{7}$ J. Kneipp, H. Kneipp, and K. Kneipp, Chem. Soc. Rev. 37(5), 1052-1060 (2008).

${ }^{8}$ X. M. Qian and S. M. Nie, Chem. Soc. Rev. 37(5), 912-920 (2008).

${ }^{9}$ H. G. Duan, H. L. Hu, K. Kumar, Z. X. Shen, and J. K. W. Yang, ACS Nano 5(9), 7593-7600 (2011).

${ }^{10}$ L. Feng, R. Ma, Y. Wang, D. Xu, D. Xiao, L. Liu, and N. Lu, Nano Res. 8(11), 3715-3724 (2015).

${ }^{11}$ H. Im, K. C. Bantz, N. C. Lindquist, C. L. Haynes, and S. H. Oh, Nano Lett. 10(6), 2231-2236 (2010).

${ }^{12}$ J. F. Li, Y. F. Huang, Y. Ding, Z. L. Yang, S. B. Li, X. S. Zhou, F. R. Fan, W. Zhang, Z. Y. Zhou, D. Y. Wu, B. Ren, Z. L. Wang, and Z. Q. Tian, Nature 464(7287), 392-395 (2010).

${ }^{13}$ H. H. Wang, C. Y. Liu, S. B. Wu, N. W. Liu, C. Y. Peng, T. H. Chan, C. F. Hsu, J. K. Wang, and Y. L. Wang, Adv. Mater. 18(4), 491-495 (2006).

${ }^{14}$ S. Yang, W. Cai, L. Kong, and Y. Lei, Adv. Funct. Mater. 20(15), 2527-2533 (2010).

${ }^{15}$ P. Nagpal, N. C. Lindquist, S. H. Oh, and D. J. Norris, Science 325(5940), 594-597 (2009).

${ }^{16}$ K. Sakoda, Opt. Express 4(5), 167-176 (1999).

${ }^{17}$ E. C. Le Ru, E. Blackie, M. Meyer, and P. G. Etchegoin, J. Phys. Chem. C 111(37), 13794-13803 (2007). 